\title{
Maximal flat antichains of minimum weight
}

\author{
Martin Grüttmüller \\ Hochschule für Technik, Wirtschaft und Kultur Leipzig \\ Fakultät Informatik, Mathematik und Naturwissenschaften \\ Gustav-Freytag-Str. 42 a \\ 04277 Leipzig, Germany \\ gruettmueller@imn.htwk-leipzig.de \\ Sven Hartmann \\ Technische Universität Clausthal \\ Institut für Informatik \\ Julius-Albert-Str. 4 \\ 38678 Clausthal-Zellerfeld, Germany \\ sven.hartmann@tu-clausthal.de \\ Uwe Leck \\ University of Wisconsin-Superior \\ Dept. of Mathematics and Computer Science \\ Belknap \& Catlin POB 2000 \\ Superior, WI 54880, USA \\ uwe.leck@uwsuper. edu

\section{Thomas Kalinowski} \\ Universität Rostock \\ Institut für Mathematik \\ Universitätsplatz 1 \\ 18051 Rostock, Germany \\ thomas.kalinowski@uni-rostock.de \\ Ian T. Roberts \\ Charles Darwin University \\ School of Engineering and IT \\ Darwin 0909, Australia \\ ian.roberts@cdu.edu.au
}

Submitted: Jun 25, 2005; Accepted: May 22, 2009; Published: May 29, 2009

Mathematics Subject Classification: 05D05, 06A07

\begin{abstract}
We study maximal families $\mathcal{A}$ of subsets of $[n]=\{1,2, \ldots, n\}$ such that $\mathcal{A}$ contains only pairs and triples and $A \nsubseteq B$ for all $\{A, B\} \subseteq \mathcal{A}$, i.e. $\mathcal{A}$ is an antichain. For any $n$, all such families $\mathcal{A}$ of minimum size are determined. This is equivalent to finding all graphs $G=(V, E)$ with $|V|=n$ and with the property that every edge is contained in some triangle and such that $|E|-|T|$ is maximum, where $T$ denotes the set of triangles in $G$. The largest possible value of $|E|-|T|$ turns out to be equal to $\left\lfloor(n+1)^{2} / 8\right\rfloor$. Furthermore, if all pairs and triples have weights $w_{2}$ and $w_{3}$, respectively, the problem of minimizing the total weight $w(\mathcal{A})$ of $\mathcal{A}$ is considered. We show that $\min w(\mathcal{A})=\left(2 w_{2}+w_{3}\right) n^{2} / 8+o\left(n^{2}\right)$ for $3 / n \leq w_{3} / w_{2}=: \lambda=\lambda(n)<2$. For $\lambda \geq 2$ our problem is equivalent to the $(6,3)$-problem of Ruzsa and Szemerédi, and by a result of theirs it follows that $\min w(\mathcal{A})=w_{2} n^{2} / 2+o\left(n^{2}\right)$.
\end{abstract}




\section{Introduction}

Let $n \geq 2$ be an integer and $[n]:=\{1,2, \ldots, n\}$. By $2^{[n]}$ we denote the family of all subsets of $[n]$ and by $\left(\begin{array}{c}{[n]} \\ k\end{array}\right)$ the family of all $k$-subsets of $[n]$. A family $\mathcal{A} \subseteq 2^{[n]}$ is an antichain if $A \nsubseteq B$ for all $\{A, B\} \subseteq \mathcal{A}$, and the antichain $\mathcal{A}$ is called flat if

$$
\mathcal{A} \subseteq\left(\begin{array}{c}
{[n]} \\
k-1
\end{array}\right) \cup\left(\begin{array}{c}
{[n]} \\
k
\end{array}\right)
$$

for some $1 \leq k \leq n$. The volume of $\mathcal{F} \subseteq 2^{[n]}$ is defined by $v(\mathcal{F}):=\sum_{F \in \mathcal{F}}|F|$.

Our interest in flat antichains is motivated mainly by the Flat Antichain Theorem which says that for every antichain $\mathcal{A} \subseteq 2^{[n]}$ there is a flat antichain $\mathcal{A}^{\prime}$ with $\left|\mathcal{A}^{\prime}\right|=|\mathcal{A}|$ and $v\left(\mathcal{A}^{\prime}\right)=v(\mathcal{A})$. This remarkable fact follows from results of Lieby [5] (see also [6]) and Kisvölcsey [4]. We define an equivalence relation on the set of all antichains in $2^{[n]}$ saying that two antichains $\mathcal{A}$ and $\mathcal{A}^{\prime}$ are equivalent if and only if $|\mathcal{A}|=\left|\mathcal{A}^{\prime}\right|$ and $v(\mathcal{A})=v\left(\mathcal{A}^{\prime}\right)$.

Given a weight function $w:\{0\} \cup[n] \mapsto \mathbb{R}^{+}$, the weight of a family $\mathcal{F} \subseteq 2^{[n]}$ is $w(\mathcal{F}):=\sum_{F \in \mathcal{F}} w(|F|)$. Griggs [2] observed that within their equivalence classes the flat antichains have minimum BLYM-values $w_{\mathrm{BLYM}}(\mathcal{F}):=\sum_{F \in \mathcal{F}}\left(\begin{array}{c}n \\ |F|\end{array}\right)^{-1}$. More generally, if the sequence $(w(i))_{i=0}^{n}$ is convex (concave), then they have minimum (maximum) weight within their equivalence classes (Hartmann, Leck and Roberts [3]).

In this paper, we study the following question: Given $1 \leq k \leq n$ and $w_{k-1}, w_{k} \in$ $\mathbb{R}^{+}$, what is the minimum weight $w(\mathcal{A})=w_{k-1}\left|\mathcal{A}_{k-1}\right|+w_{k}\left|\mathcal{A}_{k}\right|$ of a maximal antichain $\mathcal{A}=\mathcal{A}_{k-1} \cup \mathcal{A}_{k}$ with $\mathcal{A}_{i} \subseteq\left(\begin{array}{c}{[n]} \\ i\end{array}\right), i=k-1, k$ ? (By maximal we mean that for any $A \in \mathcal{A} \backslash\left(\left(\begin{array}{c}{[n]} \\ k-1\end{array}\right) \cup\left(\begin{array}{c}{[n]} \\ k\end{array}\right)\right)$ the family $\mathcal{A} \cup\{A\}$ is not an antichain.) In [3], the same problem has been solved under the additional constraint that $\mathcal{A}$ is squashed, i.e. $\mathcal{A}_{k}$ is an initial segment of $\left(\begin{array}{c}{[n]} \\ k\end{array}\right)$ with respect to the colexicographic order. If $k \leq 2$, then any $\mathcal{A}$ can be transformed into a squashed $\mathcal{A}^{\prime}$ by an appropriate permutation of $[n]$. Trivially, for $k=1$, the smallest possible $w(\mathcal{A})$ is $w_{0}$ if $w_{0} / w_{1} \leq n$ and $n w_{1}$ otherwise. For $k=2$, it is an easy exercise to show that it is best possible to choose $\left|\mathcal{A}_{1}\right|=n$ if $w_{1} / w_{2}<1 / 2$, $\left|\mathcal{A}_{1}\right| \in\{n-2, n\}$ if $w_{1} / w_{2}=1 / 2,\left|\mathcal{A}_{1}\right|=n-2$ if $1 / 2<w_{1} / w_{2} \leq 1$, and $\left|\mathcal{A}_{1}\right|$ equal to one of the non-negative integers closest to $n-1 / 2-w_{1} / w_{2}$ if $w_{1} / w_{2}>1$.

For the rest of the paper, we concentrate on the case $k=3$. Without loss of generality, we put $w_{2}=1$ and $w_{3}=\lambda$. Let $\mathcal{A}=\mathcal{A}_{2} \cup \mathcal{A}_{3} \subseteq 2^{[n]}$ be a maximal antichain with $\mathcal{A}_{i} \subseteq\left(\begin{array}{c}{[n]} \\ i\end{array}\right)$ for $i=2,3$. With $\mathcal{A}$ we associate a graph $G(\mathcal{A})$ on $[n]$ defined by

$$
E(G(\mathcal{A}))=\left(\begin{array}{c}
{[n]} \\
2
\end{array}\right) \backslash \mathcal{A}_{2}
$$

By the maximality of $\mathcal{A}$, every edge from $E$ is a subset of some set from $\mathcal{A}_{3}$ and $\mathcal{A}_{3}$ is the set of all triangles in $G(\mathcal{A})$. Hence, for $\lambda \in \mathbb{R}^{+}$the optimization problem

$$
w(\mathcal{A}):=\left|\mathcal{A}_{2}\right|+\lambda\left|\mathcal{A}_{3}\right| \rightarrow \min
$$

is equivalent to the problem

$$
|E|-\lambda|T| \rightarrow \max
$$


where the optimization is over all graphs $G=(V, E)$ with $|V|=n$ and the property that every edge from $E$ is contained in at least one triangle from $T$, the set of all triangles in $G$. In the sequel, graphs with this property will be called $T$-graphs.

For $x \in V$, let $N(x):=\{y \in V: x y \in E\}$ and $\bar{N}(x):=N(x) \cup\{x\}$. Furthermore, for $x \in V \cup E$ let $D(x)$ denote the number of triangles in $T$ containing $x$.

Throughout, the sets of vertices, edges and triangles of a graph $G$ will be denoted by $V, E$ and $T$, respectively, and $d(i)$ is the degree of vertex $i$.

\section{The bound}

Theorem 1. Let $G$ be a T-graph on $n$ vertices. Then

$$
|E|-\lambda|T| \leq \frac{(n+\lambda)^{2}}{8 \lambda}
$$

holds for all positive real numbers $\lambda$.

Proof. Fix some $x y z \in T$, and for $i=0,1,2,3$ let $a_{i}$ be the number of vertices $v \in$ $V \backslash\{x, y, z\}$ with $|N(v) \cap\{x, y, z\}|=i$. Then

$$
\begin{aligned}
a_{0}+a_{1}+a_{2}+a_{3} & =n-3, \\
a_{1}+2 a_{2}+3 a_{3} & =d(x)+d(y)+d(z)-6, \\
a_{2}+3 a_{3} & =D(x y)+D(y z)+D(x z)-3,
\end{aligned}
$$

and consequently,

$$
d(x)+d(y)+d(z)-D(x y)-D(y z)-D(x z)-3=a_{1}+a_{2} \leq n-3 .
$$

Hence, there are nonnegative integers $\alpha_{x y z}(x y z \in T)$ such that

$$
d(x)+d(y)+d(z)=n+3+(D(x y)-1)+(D(y z)-1)+(D(x z)-1)-\alpha_{x y z}
$$

for all $x y z \in T$. Summing up (2) over $T$ yields

$$
\sum_{x \in V} D(x) d(x)=(n+3)|T|+\sum_{x y \in E} D(x y)(D(x y)-1)-\alpha,
$$

where

$$
\alpha=\sum_{x y z \in T} \alpha_{x y z}
$$

For all $x \in V$ the equation

$$
D(x)=\frac{1}{2} \sum_{y: x y \in E} D(x y)=\frac{1}{2} d(x)+\frac{1}{2} \sum_{y: x y \in E}(D(x y)-1) .
$$


holds. Substituting into (3) yields

$$
\begin{array}{r}
\frac{1}{2} \sum_{x \in V} d(x)^{2}+\sum_{x y \in E}(D(x y)-1)\left(\frac{d(x)+d(y)}{2}-1\right)+\sum_{x y \in E}(D(x y)-1) \\
=(n+3)|T|+\sum_{x y \in E} D(x y)(D(x y)-1)-\alpha .
\end{array}
$$

Clearly,

$$
D(x y)=|N(x) \cap N(y)| \leq \min \{d(x), d(y)\}-1 \leq \frac{d(x)+d(y)}{2}-1
$$

for all $x y \in E$. Define

$$
\beta_{x y}:=(D(x y)-1)\left(\frac{d(x)+d(y)}{2}-1-D(x y)\right)
$$

for all $x y \in E$. Note that $\beta_{x y} \geq 0$ as $D(x y) \geq 1$ for all $x y \in E$. By (4) we have

$$
\frac{1}{2} \sum_{x \in V} d(x)^{2}+3|T|-|E|=(n+3)|T|-\alpha-\beta,
$$

where

$$
\beta=\sum_{x y \in E} \beta_{x y}
$$

For $x \in V$, put

$$
\gamma_{x}:=\frac{n+\lambda}{2 \lambda}-d(x)
$$

Then

$$
d(x)^{2}=\left(\frac{n+\lambda}{2 \lambda}\right)^{2}-2 \frac{n+\lambda}{2 \lambda} \gamma_{x}+\gamma_{x}^{2}
$$

and with $\sum_{x \in V} \gamma_{x}=n \frac{n+\lambda}{2 \lambda}-2|E|$,

$$
\sum_{x \in V} d(x)^{2}=n\left(\frac{n+\lambda}{2 \lambda}\right)^{2}-2 n\left(\frac{n+\lambda}{2 \lambda}\right)^{2}+4 \frac{n+\lambda}{2 \lambda}|E|+\gamma
$$

where

$$
\gamma=\sum_{x \in V} \gamma_{x}^{2}
$$

Substituting (8) into (6) yields

$$
\frac{n}{\lambda}|E|-n|T|=\frac{n}{2}\left(\frac{n+\lambda}{2 \lambda}\right)^{2}-\alpha-\beta-\frac{\gamma}{2} .
$$


Hence,

$$
|E|-\lambda|T|=\frac{\lambda}{2}\left(\frac{n+\lambda}{2 \lambda}\right)^{2}-\frac{\lambda}{n}\left(\alpha+\beta+\frac{\gamma}{2}\right),
$$

and (1) follows by $\alpha, \beta, \gamma \geq 0$.

Corollary 2. If $\mathcal{A} \subseteq\left(\begin{array}{c}{[n]} \\ 2\end{array}\right) \cup\left(\begin{array}{c}{[n]} \\ 3\end{array}\right)$ is a maximal antichain, then

$$
w(\mathcal{A}) \geq\left(\begin{array}{l}
n \\
2
\end{array}\right)-\frac{(n+\lambda)^{2}}{8 \lambda} .
$$

Obviously, the quality of the bound (1) depends on $\lambda$. The bound (1) is best possible for $\lambda=1$, as will be shown in the next section, whereas for $\lambda \leq 1 / 4$ it is worse than the trivial upper bound $\left(\begin{array}{l}n \\ 2\end{array}\right) .|E| \leq 3|T|$ implies that for $\lambda \geq 3$ it is best to choose $G$ to be the empty graph. For $\lambda \leq 1 /(n-2)$ it is clear that $|E|-\lambda|T|$ is maximized when $G=K_{n}$. Some improvements of (1) for $1 /(n-2)<\lambda<3, \lambda \neq 1$ are given in Sections 4 and 5 .

\section{Maximal flat antichains of minimum size}

In this section we show that the bound (11) is tight for $\lambda=1$ and construct all antichains for which it is attained. Obviously, this is equivalent to finding all T-graphs on $n$ vertices for which equality holds in Theorem 1, i.e. for which $|E|-|T|$ becomes a maximum. The parallels to a very basic problem in extremal graph theory are evident: Find all trianglefree graphs on $n$ vertices with the largest possible number of edges. The solution to this is of course well-known, with complete bipartite graphs with (almost) equal bipartition sets being optimal. A way of looking at our problem is the following: We also want to have many edges but we have to "pay" for the triangles. Some triangles are clearly unavoidable as we are considering T-graphs but a possible approach is to start with complete bipartite graphs and add just as many edges as are necessary to make sure that every edge is contained in some triangle. It will turn out that, in general, the T-graphs solving our problem are of this kind. However, for some small values of $n$, there are also other "sporadic" optimal T-graphs. This might be an indication that in proving that for large enough $n$ the modified complete bipartite graphs are the only solutions, a good amount of technicalities and case studies will be unavoidable.

For positive integers $n, s, 0<2 s<n$, let $K_{2 s, n-2 s}^{+}$denote the graph on $[n]$ with edge set

$$
E\left(K_{2 s, n-2 s}^{+}\right)=([2 s] \times([n] \backslash[2 s])) \cup\{\{i, i+s\}: i=1,2, \ldots, s\},
$$

see Figure 1 for an illustration. Furthermore, let $G_{9}$ denote the graph on $\mathbb{Z}_{3} \times \mathbb{Z}_{3}$ with edge set

$$
E\left(G_{9}\right)=\{\{(x, y),(u, v)\}: x \neq u, y \neq v\},
$$

see Figure 2, and let $G_{5 a}$ and $G_{5 b}$ be the graphs displayed in Figures 3 and 4, respectively. 


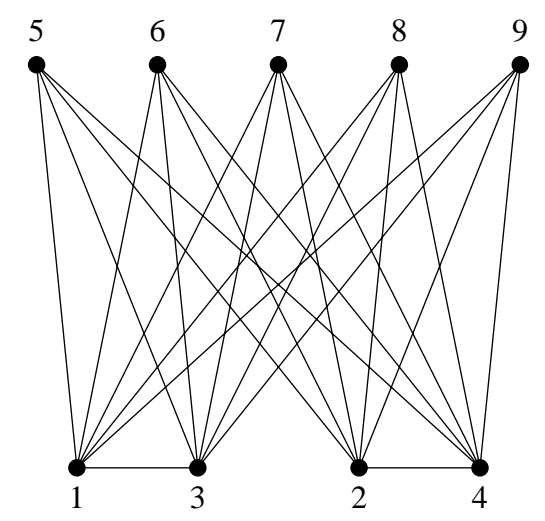

Figure 1: The graph $K_{4,5}^{+}$

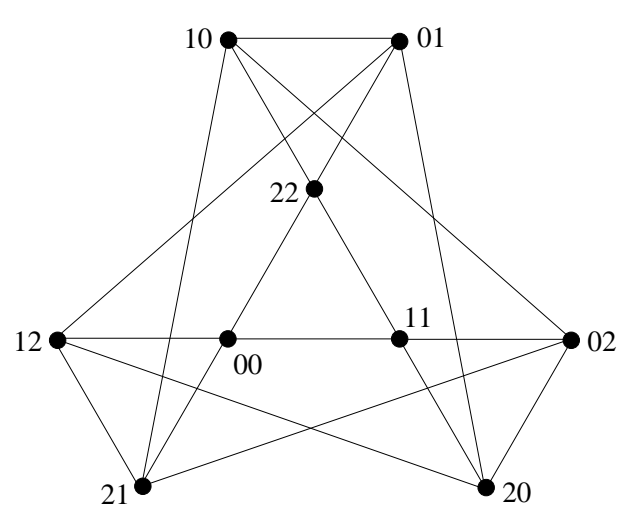

Figure 2: The graph $G_{9}$

Theorem 3. Let $\mathcal{A} \subseteq\left(\begin{array}{c}{[n]} \\ 2\end{array}\right) \cup\left(\begin{array}{c}{[n]} \\ 3\end{array}\right)$ be a maximal antichain. Then

$$
|\mathcal{A}| \geq\left(\begin{array}{l}
n \\
2
\end{array}\right)-\left\lfloor\frac{(n+1)^{2}}{8}\right\rfloor,
$$

and equality holds if and only if

(i) $n \equiv 0(\bmod 4)$ and $G(\mathcal{A}) \cong K_{n / 2, n / 2}^{+}$, or

(ii) $n \equiv 1(\bmod 4)$ and $G(\mathcal{A}) \cong K_{(n-1) / 2,(n+1) / 2}^{+}$

or $G(\mathcal{A}) \cong K_{(n+3) / 2,(n-3) / 2}^{+}$

or $\quad G(\mathcal{A}) \cong G_{5 a}$

or $G(\mathcal{A}) \cong G_{5 b}$

or $G(\mathcal{A}) \cong G_{9}$, or

(iii) $n \equiv 2(\bmod 4)$ and $G(\mathcal{A}) \cong K_{(n+2) / 2,(n-2) / 2}^{+}$, or

(iv) $n \equiv 3(\bmod 4)$ and $G(\mathcal{A}) \cong K_{(n+1) / 2,(n-1) / 2}^{+}$.

Proof. The inequality (12) follows immediately from Corollary 2, and it is obvious that equality holds if and only if

$$
|E|-|T|=\left\lfloor\frac{(n+1)^{2}}{8}\right\rfloor
$$

for $G=G(\mathcal{A})$. If $G=G(\mathcal{A})$ is like in (i)-(iv), then equality holds in (13).

Let $G$ be some T-graph on $n$ vertices such that (13) is satisfied. It remains to show that $G$ is isomorphic to one of the graphs listed in the theorem.

According to (10) in the proof of Theorem 1, (13) is equivalent to 


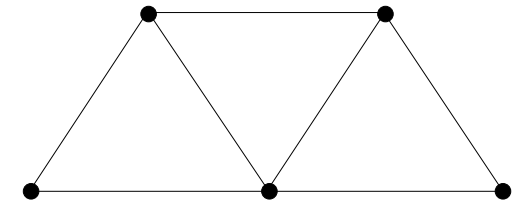

Figure 3: The graph $G_{5 a}$

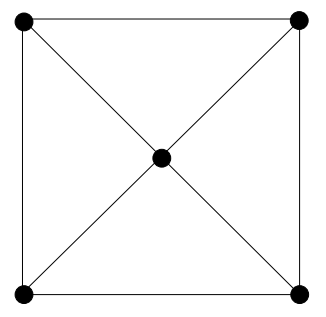

Figure 4: The graph $G_{5 b}$

$$
2(\alpha+\beta)+\gamma=\varepsilon n \quad \text { with } \varepsilon:=\left\{\begin{array}{lll}
1 / 4 & \text { if } n \equiv 0 & (\bmod 2), \\
1 & \text { if } n \equiv 1 & (\bmod 4), \\
0 & \text { if } n \equiv 3 & (\bmod 4)
\end{array}\right.
$$

Recall that

$$
\alpha=\sum_{x y z \in T} \alpha_{x y z}, \quad \beta=\sum_{x y \in E} \beta_{x y}, \quad \gamma=\sum_{x \in V} \gamma_{x}^{2},
$$

where $\alpha_{x y z}, \beta_{x y}, \gamma_{x}$ are defined in (2), (5) and (7), respectively, and the numbers $\alpha_{x y z}, \beta_{x y}$ are non-negative. Note that $\alpha_{x y z}$ is equal to the number of vertices in $V$ that are adjacent to none or to all of the vertices $x, y, z$. Further note that

$$
\beta_{x y}=0 \quad \Longleftrightarrow \quad D(x y)=1 \text { or } \bar{N}(x)=\bar{N}(y) .
$$

The proof is by induction on $n$. Using (14), it is easy to show that $G$ is isomorphic to one of the graphs listed in the theorem if $n \leq 9$. In the sequel, we assume $n \geq 10$ and that the assertion is true for all T-graphs $G^{\prime}$ on $n^{\prime}<n$ vertices.

Case 1: $n \equiv 0(\bmod 2)$.

By (7), we have $\left|\gamma_{x}\right| \geq 1 / 2$ for all $x$. Hence, $\gamma \geq n / 4$, and (14) yields $\gamma=n / 4$ and

$$
\begin{aligned}
\forall x \in V: & d(x) \in\{n / 2, n / 2+1\}, \\
\forall x y \in E: & \beta_{x y}=0, \\
\forall x y z \in T: & \alpha_{x y z}=0 .
\end{aligned}
$$

Using (17), (2) becomes $d(x)+d(y)+d(z)=n+D(x y)+D(y z)+D(x z)$, and with (15) we obtain

$$
\forall x y z \in T: \quad \frac{n}{2} \leq D(x y)+D(x z)+D(y z) \leq \frac{n}{2}+3,
$$

and by (5), (15) and (16),

$$
\forall x y \in E: \quad D(x y)=1 \quad \text { or } \quad D(x y) \in\{n / 2-1, n / 2\} .
$$


(18) and (19) imply that for every triangle $x y z \in T$ there is a permutation $\pi$ of its edges such that

$$
D(\pi(x y))=D(\pi(x z))=1, \quad D(\pi(y z)) \in\{n / 2-1, n / 2\} .
$$

Hence, for every $y z \in E$ with $D(y z)>1, T$ contains $D(y z) \in\{n / 2-1, n / 2\}$ triangles $x_{i} y z$ with $D\left(x_{i} y\right)=D\left(x_{i} z\right)=1, i=1,2, \ldots, D(y z)$.

Case 1.1: $n \equiv 0(\bmod 4)$.

Assume that $D(y z)=n / 2-1$ for some $y z \in E$. Let $G^{\prime}$ be the T-graph obtained by deleting all triangles containing $y z$ as an edge and the vertices $y, z$ from $G$. Then $G^{\prime}$ is a graph on $n-2$ vertices with $\left|E^{\prime}\right|-\left|T^{\prime}\right|=\left\lfloor(n-1)^{2} / 8\right\rfloor$, and, by induction, $G^{\prime} \cong K_{n / 2,(n-4) / 2}^{+}$. Therefore, there are $n / 2$ vertices of degree $n / 2-1$ in $G^{\prime}$. All these vertices must have been adjacent to $y$ and $z$ in $G$ because of (15). Hence, $D(y z) \geq n / 2$, contradicting our assumption.

Consequently, $D(x y) \in\{1, n / 2\}$ holds for all $x y \in E$ and thereby

$$
\forall x y z \in T: \quad D(x y)+D(x z)+D(y z)=\frac{n}{2}+2 .
$$

By (15), (17), (20), two vertices of any triangle from $T$ have degree $n / 2+1$ while its third vertex has degree $n / 2$. Clearly, $D(y z)=n / 2$ implies $d(y)=d(z)=n / 2+1$. Let $G^{\prime}$ be obtained from $G$ by deleting a vertex $x$ with $d(x)=n / 2$ and all edges incident with $x$. Then $G^{\prime}$ is a T-graph on $n-1$ vertices with $\left|E^{\prime}\right|-\left|T^{\prime}\right|=n^{2} / 8$, and, by induction, $G^{\prime} \cong K_{n / 2,(n-2) / 2}^{+}$. Hence, there are exactly $n / 4$ edges which are contained in more than one triangle in $G^{\prime}$, and these edges form a matching in $G^{\prime}$. The end-vertices of these edges must form the neighborhood of $x$ in $G$, i.e. $G \cong K_{n / 2, n / 2}^{+}$.

Case 1.2: $n \equiv 2(\bmod 4)$.

Assume that $D(y z)=n / 2-1$ for some $y z \in E$. Let $G^{\prime}$ be the T-graph obtained by deleting all triangles containing $y z$ as an edge and the vertices $y, z$ from $G$. Then $G^{\prime}$ is a graph on $n-2$ vertices with $\left|E^{\prime}\right|-\left|T^{\prime}\right|=\left\lfloor(n-1)^{2} / 8\right\rfloor$, and, by induction, $G^{\prime} \cong K_{(n-2) / 2,(n-2) / 2}^{+}$. Therefore, there are $n / 2-1$ vertices of degree $n / 2-1$ in $G^{\prime}$. All these vertices must have been adjacent to $y$ and $z$ in $G$ because of $(15)$. Hence, $G \cong K_{(n+2) / 2,(n-2) / 2}^{+}$.

Consequently, w.l.o.g. we can assume that $D(x y) \in\{1, n / 2\}$ for all $x y \in E$. As in Case 1.1, every triangle contains a vertex of degree $n / 2$. Let $x \in V$ with $d(x)=n / 2$. Then $D(x y)=1$ must hold for all $x y \in E$. Hence, the degree of $x$ is even, a contradiction.

Case 2: $n \equiv 1(\bmod 4)$.

Let

$$
M:=\{x y \in E: \bar{N}(x)=\bar{N}(y)\} .
$$

Note that by (5), $\beta_{x y}=0$ for all $x y \in M$.

Claim 1: $\quad M \neq \varnothing$.

Proof: Assume that $M=\varnothing$. Then

$$
2 \beta \geq \sum_{x y \in E}(D(x y)-1)=3|T|-|E|,
$$


where the inequality follows from (5). Furthermore,

$$
\gamma=\sum_{x \in V}\left(\frac{n+1}{2}-d(x)\right)^{2} \geq \sum_{x \in V}\left(\frac{n+1}{2}-d(x)\right)
$$

implies

$$
\gamma \geq \frac{n(n+1)}{2}-2|E|
$$

By (14), (21), (22), (12),

$$
n \geq 2 \beta+\gamma \geq \frac{n(n+1)}{2}+3|T|-3|E|=\frac{n^{2}-2 n+9}{8}
$$

which yields $n \leq 9$.

For $x y \in E$ put $V_{1}(x y):=\{z \in V: x y z \in T\}$, and let

$$
E\left(V_{1}(x y)\right):=\left\{u v \in E: u, v \in V_{1}(x y)\right\} .
$$

Claim 2: W.l.o.g. we can assume that

$$
\forall x y \in M: \quad d(x)-\left|E\left(V_{1}(x y)\right)\right| \geq \frac{n+1}{2} .
$$

Proof: Let $x y \in M$, and let $G^{\prime}$ be obtained from $G$ by removing $x, y$, and all edges $u v \in E\left(V_{1}(x y)\right)$ with $D(u v)=2$. Then $G^{\prime}$ is a T-graph on $n-2$ vertices with

$$
\begin{aligned}
\left|E^{\prime}\right|-\left|T^{\prime}\right|= & \frac{n^{2}+2 n-3}{8}-d(x) \\
& \quad-\left|\left\{u v \in E\left(V_{1}(x y)\right): D(u v)=2\right\}\right|+2\left|E\left(V_{1}(x y)\right)\right| \\
\geq & \frac{n^{2}+2 n-3}{8}-\left(d(x)-\left|E\left(V_{1}(x y)\right)\right|\right) .
\end{aligned}
$$

Together with $\left|E^{\prime}\right|-\left|T^{\prime}\right| \leq(n-1)^{2} / 8$, which follows from Theorem 1 , we obtain

$$
d(x)-\left|E\left(V_{1}(x y)\right)\right| \geq \frac{n-1}{2} .
$$

Assume that equality holds in (23). Then, by induction, $G^{\prime} \cong K_{(n-1) / 2,(n-3) / 2}^{+}$. Moreover, $D(u v)=2$ must hold for all $u v \in E\left(V_{1}(x y)\right)$. Hence, $V_{1}(x y)$ is the unique independent set of size $(n-3) / 2$ in $G^{\prime}$. But then, for every $u v \in E$ with $u, v \in V_{1}(x y)$ we have $D(u v)=(n+3) / 2>2$. So $V_{1}(x y)$ must be independent also in $G$. Consequently, $d(x)=(n-1) / 2$ which eventually leads to $G \cong K_{(n+3) / 2,(n-3) / 2}^{+}$. 
Claim 3: $V_{1}(x y)$ is an independent set for all $x y \in M$.

Proof: Assume that $x y \in M$ and $u v \in E\left(V_{1}(x y)\right)$. Then

$$
D(x u), D(x v), D(y u), D(y v) \geq 2,
$$

and by (5),

$$
\begin{aligned}
\beta & \geq \beta_{x u}+\beta_{x v}+\beta_{y u}+\beta_{y v} \\
& \geq\left(\frac{d(x)+d(u)}{2}-1-D(x u)\right)+\cdots+\left(\frac{d(y)+d(v)}{2}-1-D(y v)\right) .
\end{aligned}
$$

We claim that each of the four summands is at least $(n-3) / 4$, hence $2 \beta \geq 2(n-3)>$ $n$, in contradiction to (14).

By $d(u) \geq D(x u)+1$, we have

$$
\frac{d(x)+d(u)}{2}-1-D(x u) \geq \frac{d(x)-D(x u)-1}{2} .
$$

On the other hand, $D(x u) \leq\left|E\left(V_{1}(x y)\right)\right|+1$, so with Claim 2,

$$
D(x u) \leq d(x)-\frac{n-1}{2}
$$

holds. Thus,

$$
\frac{d(x)+d(u)}{2}-1-D(x u) \geq \frac{n-3}{4} .
$$

Analogously, each of the other summands is at least $(n-3) / 4$.

By Claims 2 and 3, the edges $M$ form a matching in $G$ and $2|M| \leq(n+1) / 2$. Since $(n+1) / 2$ is odd, we conclude $|M| \leq(n-1) / 4$. Assume that $|M|=(n-1) / 4$. Let $V(M)$ denote the set of vertices incident with an edge in $M$. By Claims 2 and 3, $G \backslash V(M)$ does not contain a triangle. If there was an edge in $G \backslash V(M)$, then the two endpoints would have a common neighbor in $V(M)$, a contradiction to Claim 3. Hence, $V \backslash V(M)$ is an independent set. Now it is easy to see that $|E|-|T|$ becomes maximum only if $G \cong K_{(n-1) / 2,(n+1) / 2}^{+}$.

In the sequel, we assume that $|M|<(n-1) / 4$.

Let

$$
\begin{aligned}
V_{1} & :=\bigcup_{x y \in M} V_{1}(x y), \\
V_{1}^{\prime} & :=\bigcap_{x y \in M} V_{1}(x y), \\
V_{2} & :=V \backslash\left(V_{1} \cup V(M)\right),
\end{aligned}
$$




$$
\begin{aligned}
E_{1} & :=\left\{x y \in E: x \in V_{1}, y \in V_{2}\right\}, \\
E_{2} & :=\left\{x y \in E: x, y \in V_{2}\right\}, \\
d_{1}(x) & :=\left|\left\{y \in V_{1}: x y \in E\right\}\right| \quad \text { for } x \in V, \\
d_{2}(x) & :=\left|\left\{y \in V_{2}: x y \in E\right\}\right| \quad \text { for } x \in V .
\end{aligned}
$$

Claim 4: $\quad\left|V_{1}^{\prime}\right| \geq\left|V_{1}\right|-\frac{n-1}{4}$.

Proof: For $z \in V_{1} \backslash V_{1}^{\prime}$, there are $x y, x^{\prime} y^{\prime} \in M$ with $z \in V_{1}(x y)$ and $z \notin V_{1}\left(x^{\prime} y^{\prime}\right)$. Hence, by Claims 2 and 3 ,

$$
d(z) \leq n-\frac{n-1}{2}-2=\frac{n-3}{2} .
$$

Thus, by (7) and (9), $\gamma \geq 4\left|V_{1} \backslash V_{1}^{\prime}\right|$, and $\left|V_{1} \backslash V_{1}^{\prime}\right| \leq \frac{n-1}{4}$ follows by (14).

Claim 5: $\quad \frac{n-1}{2} \leq\left|V_{1}\right| \leq \frac{n+1}{2}$.

Proof: The first inequality follows from $V_{1} \supseteq V_{1}(x y)$ and $\left|V_{1}(x y)\right| \geq \frac{n-1}{2}$ for every $x y \in M$. Assume $\left|V_{1}\right| \geq \frac{n+3}{2}$. Then, by Claim $4,\left|V_{1}^{\prime}\right| \geq \frac{n+7}{4}$, and, by Claim 3 , for every $z \in V_{1}^{\prime}$ we have

hence

$$
d(z) \leq n-\left|V_{1}\right| \leq \frac{n-3}{2}
$$

$$
\gamma \geq \frac{n+7}{4} \cdot 4>n
$$

a contradiction to (14).

Claim 6: $\left|E\left(V_{1}\right)\right| \leq 1$ and $\left|E\left(V_{1}\right)\right|=\varnothing$ if $V_{1}=V_{1}(x y)$ for some $x y \in M$.

Proof: If there is some $x y \in M$ with $V_{1}=V_{1}(x y)$, this is Claim 3. So assume $\left|V_{1}\right|=\frac{n+1}{2}$ and $\left|V_{1}(x y)\right|=\frac{n-1}{2}$ for all $x y \in M$, i.e. for each $x y \in M$ there is a unique $z \in V_{1}$ such that $V_{1}(x y)=V_{1} \backslash\{z\}$. Now let $w z \in E\left(V_{1}\right)$. There are $x y, x^{\prime} y^{\prime} \in M$, such that

$$
\{w\}=V_{1} \backslash V_{1}(x y),\{z\}=V_{1} \backslash V_{1}\left(x^{\prime} y^{\prime}\right) .
$$

Then every edge in $E\left(V_{1}\right) \backslash\{w z\}$ would have both vertices in $V_{1}(x y)$ or in $V_{1}\left(x^{\prime} y^{\prime}\right)$, contradicting Claim 3.

Claim 7: If $V_{1}$ is an independent set, then $d_{2}(x) \geq 1$ for every $x \in V_{2}$ and $d_{2}(x)+d_{2}(y) \geq$ 3 for every $x y \in E_{2}$.

Proof: Let $x \in V_{2}$ with $d_{2}(x)=0$. Then also $d_{1}(x)=0$ because an edge $x y$ with $y \in V_{1}$ would not be contained in any triangle. So $d(x)=0$ and deleting $x$ we would obtain a T-graph on $n-1$ vertices violating the bound from Theorem 1 . Now assume $x y \in E_{2}$ with $d_{2}(x)=d_{2}(y)=1$. Then for $z \in V_{1}$ we have

$$
x z \in E \Longleftrightarrow y z \in E,
$$

hence $x y \in M$, a contradiction. 
Case 2.1: $\left|V_{1}\right|=\frac{n-1}{2}$.

In this case we have $x y \in E$ for every $(x, y) \in V_{1} \times V(M)$, and $V_{1}$ is an independent set. Let $\delta=\frac{n-1}{2}\left|V_{2}\right|-\left|E_{1}\right|$, i.e. the number of non-edges between $V_{1}$ and $V_{2}$. We use the following simple observations:

$$
\begin{gathered}
\alpha \geq|M| \delta, \\
\gamma \geq \sum_{x \in V_{1}}\left(\frac{n+1}{2}-d(x)\right)^{2} \geq \delta .
\end{gathered}
$$

Now from $2 \alpha+\gamma \leq n$ and $|M| \geq 1$ we obtain

$$
\delta \leq \frac{n}{3}
$$

With $\frac{n-1}{2}>\frac{n}{3}$ this implies that there is a vertex $z_{0} \in V_{1}$ with $z_{0} y \in E$ for every $y \in V_{2}$. We claim that $D(x y)>1$ for all $x y \in E_{2}$. Assume $D(x y)=1$ for some $x y \in E_{2}$. Then $z_{0}$ is the only vertex in $V_{1}$ that is adjacent with both, $x$ and $y$. So

$$
\delta \geq\left|V_{1}\right|-1=\frac{n-3}{2}
$$

and together with (24), $n \leq 9$ and the claim is proved.

Now let $G^{\prime}=\left(V^{\prime}, E^{\prime}\right)$ be the graph obtained from $G$ by deleting $z_{0}$ and all edges incident with $z_{0}$, and let $T^{\prime}$ be the set of triangles in $G^{\prime}$. $G^{\prime}$ is a T-graph on $n-1$ vertices, and by induction

$$
\left|E^{\prime}\right|-\left|T^{\prime}\right| \leq \frac{n^{2}-1}{8}
$$

With (13) and

$$
\left|E^{\prime}\right|=|E|-\frac{n+1}{2} \text { and }\left|T^{\prime}\right|=|T|-|M|-\left|E_{2}\right|
$$

we obtain

$$
\left|E_{2}\right|+|M| \leq \frac{n+3}{4},
$$

and finally with $|M|=\frac{1}{2}\left(\frac{n+1}{2}-\left|V_{2}\right|\right)$,

$$
\left|E_{2}\right| \leq \frac{\left|V_{2}\right|+1}{2}
$$

By Claim 7, $\left|E_{2}\right| \geq 2\left|V_{2}\right| / 3$, and therefore $\left|V_{2}\right| \leq 3$. In case of equality, by induction we have $G^{\prime} \cong K_{(n-1) / 2,(n-1) / 2}^{+}$. But this is impossible, because for $x \in V(M)$ its degree in $G^{\prime}$ is $(n-1) / 2$ while in $G^{\prime} \cong K_{(n-1) / 2,(n-1) / 2}^{+}$, for every edge $x y$ with $D(x y)>1$ we have $d(x)=d(y)=(n+1) / 2$. So we obtain a contradiction as $\left|V_{2}\right|$ must be odd and $\left|V_{2}\right|=1$ contradicts Claim 7. 
Case 2.2: $\left|V_{1}\right|=\frac{n+1}{2}$.

For $z \in V_{1}$ we put

$$
\begin{aligned}
\alpha_{z} & =\sum_{x y \in M: x y z \in T} \alpha_{x y z}, \\
\beta_{z} & =\sum_{y \in V_{2}: y z \in E}(D(y z)-1)\left(\frac{d(y)+d(z)}{2}-1-D(y z)\right) .
\end{aligned}
$$

Then it is easy to see that

$$
2(\alpha+\beta)+\gamma \geq \sum_{z \in V_{1}} 2\left(\alpha_{z}+\beta_{z}\right)+\gamma_{z}^{2}
$$

By (14), there must exist a $z_{0} \in V_{1}$ with

$$
2\left(\alpha_{z_{0}}+\beta_{z_{0}}\right)+\gamma_{z_{0}}^{2} \leq 1
$$

By Claim 6, $\left|E\left(V_{1}\right)\right| \leq 1$. If $d_{1}\left(z_{0}\right)=1$, then $d\left(z_{0}\right) \leq \frac{n-3}{2}$ because there are $x, y \in V(M)$ that are not adjacent to $z_{0}$ by Claim 3. Hence, $\gamma_{z_{0}} \geq 2$, a contradiction. This implies $d_{1}\left(z_{0}\right)=0$ and thereby, $d\left(z_{0}\right) \leq(n-1) / 2$. By $\gamma_{z_{0}} \leq 1$, it follows that $d\left(z_{0}\right)=(n-1) / 2$ and $\gamma_{z_{0}}=1$. Consequently, $\alpha_{z_{0}}=\beta_{z_{0}}=0$. Further, it follows that $z_{0}$ is adjacent to all $x \in V_{2} \cup V(M)$. By $\beta_{z_{0}}=0$ and the fact that there is no edge between $V(M)$ and $V_{2}$, $D\left(z_{0} x\right)=1$ holds for all $x \in V_{2}$. This implies $d_{2}(x)=1$ for all $x \in V_{2}$, and by Claim 7 it follows that $V_{1}$ is not an independent set. So assume $u v \in E$ with $u, v \in V_{1}$. Since $d_{1}\left(z_{0}\right)=0$, we have $z_{0} \notin\{u, v\}$. Now let $x y \in M$ with $x y z_{0} \in T$. We have either $x y u \notin T$ or $x y v \notin T$, implying $\alpha_{x y z_{0}} \geq 1$, contradicting $\alpha_{z_{0}}=0$.

Case 3: $n \equiv 3(\bmod 4)$.

By (14), the equations (16) and (17) hold also in this case and

$$
\forall x \in V: \quad d(x)=\frac{n+1}{2} .
$$

By (2),(17) and (25),

$$
\forall x y z \in T: \quad D(x y)+D(x z)+D(y z)=\frac{n+3}{2},
$$

and by $(5),(16)$ and $(25), D(x y) \in\{1,(n-1) / 2\}$ for all $x y \in E$. Hence, for every triangle $x y z \in T$ there is a permutation $\pi$ of its edges such that

$$
D(\pi(x y))=D(\pi(x z))=1, \quad D(\pi(y z))=\frac{n-1}{2} .
$$

Let $y z \in E$ with $D(y z)=(n-1) / 2$, and let $G^{\prime}$ be the T-graph obtained by deleting all triangles containing $y z$ as an edge and the vertices $y, z$ from $G$. Then $G^{\prime}$ is a graph on $n-2$ vertices with $\left|E^{\prime}\right|-\left|T^{\prime}\right|=\left\lfloor(n-1)^{2} / 8\right\rfloor$ and with exactly $(n-1) / 2$ vertices of degree $(n-3) / 2$. By induction, $G^{\prime} \cong K_{(n-3) / 2,(n-1) / 2}^{+}$which eventually implies $G \cong$ $K_{(n+1) / 2,(n-1) / 2}^{+}$. 


\section{The case $\lambda<2$}

For $\lambda \neq 1$ we can prove only asymptotic results. A very interesting case of the original antichain problem is the BLYM-value, which corresponds to $\lambda=3 /(n-2)$ in the T-graph formulation. This is a motivation to consider not only constant weights but also weights depending on $n$, i.e. the weight is a function $\lambda: \mathbb{N} \mapsto \mathbb{R}_{+}$, and our aim is to find for each $n \in \mathbb{N}$ a T-graph $G_{n}=\left(V_{n}, E_{n}\right)$ on $n$ vertices, such that $\left|E_{n}\right|-\lambda(n)\left|T_{n}\right|$ is maximal among all T-graphs on $n$ vertices, where $T_{n}$ is the set of triangles in $G_{n}$. For notational convenience we put

$$
\varphi_{\lambda}(n)=\max \{|E|-\lambda(n)|T|: G \text { is a T-graph on } n \text { vertices }\} .
$$

and assume throughout that $G_{n}=\left(V_{n}, E_{n}\right)$ is an optimal sequence, i.e. a sequence of T-graphs with $\left|E_{n}\right|-\lambda(n)\left|T_{n}\right|=\varphi_{\lambda}(n)$. In this section we consider the case $0<\lambda(n)<2$ for all $n$. For our standard construction $K_{2 s, n-2 s}^{+}(s=\lceil n / 4\rceil)$ we have

$$
|E|-\lambda(n)|T|=\frac{2-\lambda(n)}{8} n^{2}+o\left(n^{2}\right),
$$

and the main result of this section is that this gives the correct quadratic term for the asymptotics. For our proof we need an estimate for the maximal number of edges in a graph $G$ on $n$ vertices with the property that every edge of $G$ is contained in exactly one triangle, i.e. $D(x y)=1$ for all $x y \in E$. We observe that asymptotically this is equivalent to the $(6,3)$-problem of Ruzsa and Szemerédi [8], asking for the maximal cardinality of a triple system on $n$ points such that there are no six points which span three triples. We consider $T$ as a triple system on $V$. In [8] it is shown that, for large $n$, in order to solve the $(6,3)$-problem we can assume that every pair $x y$ is contained in at most one triple. Under this assumption, it is easy to see, that $D(x y)=1$ for every $x y \in E$ is even equivalent to the condition of the $(6,3)$-problem: Assume that $D(x y)=1$ for every $x y \in E$ and that there are three triples on at most six vertices in $T$. Clearly, two of these triples must share (exactly) one vertex. Let these triples be $x y z$ and $x u v$. Since the third triple can share at most one vertex with either of those, without loss of generality, we can assume that it is $y u w$ (with $w \notin\{x, z, v\}$ ). This implies $x y, x u, y u \in E$, hence $x y u \in T$ and $D(x y) \geq 2$, a contradiction.

Ruzsa and Szemerédi [8] use the Regularity Lemma to prove that $|T|$ is $o\left(n^{2}\right)$. We use this in the proof of the following theorem.

Theorem 4. For every $\lambda: \mathbb{N} \mapsto(0,2)$ with $\lambda(n) \geq 3 / n$ for sufficiently large $n$, we have

$$
\varphi_{\lambda}(n)=\frac{2-\lambda(n)}{8} n^{2}+o\left(n^{2}\right)
$$

Proof. $\varphi_{\lambda}(n) \geq \frac{2-\lambda(n)}{8} n^{2}+o\left(n^{2}\right)$ follows from the objective values for the $K_{2 s, n-2 s}^{+}$. Assume the statement of the theorem is false. Then there exists $\varepsilon>0$ and an infinite subset $M \subseteq \mathbb{N}$ such that

$$
\forall n \in M:\left|E_{n}\right|-\lambda(n)\left|T_{n}\right| \geq\left(\frac{2-\lambda(n)}{8}+\varepsilon\right) n^{2} .
$$


Claim 1: For $n \in M$, we have $\left|E_{n}\right| \leq n^{2} / 4+o\left(n^{2}\right)$.

Proof: If the claim is false, we have an $\varepsilon^{\prime}>0$ and an infinite set $M^{\prime} \subseteq M$ such that

$$
\forall n \in M^{\prime}:\left|E_{n}\right| \geq\left(1 / 4+\varepsilon^{\prime}\right) n^{2} .
$$

W.l.o.g. we assume $\varepsilon=\varepsilon^{\prime}$. We will show that this implies $\left|E_{n}\right| \geq n^{2}$ for sufficiently large $n$, which clearly is impossible. Split $M$ into two (possibly empty) subsets $M_{1}$ and $M_{2}$,

$$
M_{1}=\{n \in M: \lambda(n) / 8<\varepsilon / 2\}, \quad M_{2}=M \backslash M_{1} .
$$

Now we define an infinite subset $M_{0}$ of $M$ :

$$
M_{0}:= \begin{cases}M_{1} \cap M^{\prime} & \text { if }\left|M_{2} \cap M^{\prime}\right| \text { is finite } \\ M_{2} \cap M^{\prime} & \text { otherwise. }\end{cases}
$$

Case 1: $M_{0} \subseteq M_{1}$. We show by induction on $k$,

$$
\forall k \in \mathbb{N}: \quad\left|E_{n}\right| \geq\left(\frac{1}{4}+\frac{k}{2} \varepsilon\right) n^{2} \quad \text { for sufficiently large } n \in M_{0} .
$$

For $k \leq 2$ the statement is true for all $n \in M_{0}$. Let $k \geq 2$, and assume the statement is true for $k$. By Moon and Moser [7], the number of triangles in a graph with $v$ vertices and $e$ edges is at least $e\left(4 e-v^{2}\right) /(3 v)$. With $\left|E_{n}\right| \geq(1 / 4+k \varepsilon / 2) n^{2}$ this implies,

$$
\left|T_{n}\right| \geq \frac{\varepsilon\left(k / 2+k^{2} \varepsilon\right) n^{3}}{3} \geq \frac{k \varepsilon}{6} n^{3} .
$$

Using the hypothesis, we obtain $\lambda(n)\left|T_{n}\right| \geq\left(k \varepsilon n^{2}\right) / 2$ for sufficiently large $n \in M_{0}$. Now from the definition of $M_{1}$ and (26) it follows that

$$
\left|E_{n}\right| \geq\left(\frac{2-\lambda(n)}{8}+\varepsilon\right) n^{2}+\lambda(n)\left|T_{n}\right| \geq\left(\frac{1}{4}+\frac{k+1}{2} \varepsilon\right) n^{2},
$$

and this concludes the argument.

Case 2: $M_{0} \subseteq M_{1}$. As in case $1,\left|E_{n}\right| \geq(1 / 4+\varepsilon) n^{2}$ implies $\left|T_{n}\right| \geq \varepsilon n^{3} / 3$, and with $\lambda(n) \geq 4 \varepsilon, \lambda(n)\left|T_{n}\right| \geq\left(4 \varepsilon^{2} n^{3}\right) / 3$, and together with (26), $\left|E_{n}\right| \geq n^{2}$ for large $n$.

Claim 2: For $n \in \mathbb{N}$, we have $\left|T_{n}\right| \geq\left|E_{n}\right| / 2+o\left(n^{2}\right)$.

Proof: For each $n$, let $T_{n}^{0} \subseteq T_{n}$ be a subset which is maximal with respect to the property that two distinct elements of $T_{n}^{0}$ do not have an edge in common, and let $E_{n}^{0}$ be the set of edges of the triangles in $T_{n}^{0}$. Then $\left|E_{n}^{0}\right|=3\left|T_{n}^{0}\right|=o\left(n^{2}\right)$ by [8]. By maximality of $T_{n}^{0}$, each of the triangles in $T_{n}^{1}:=T_{n} \backslash T_{n}^{0}$ has at least on edge in $E_{n}^{0}$. With $E_{n}^{1}:=E_{n} \backslash E_{n}^{0}$, this implies $\left|T_{n}^{1}\right| \geq\left|E_{n}^{1}\right| / 2$, and with $\left|T_{n}\right|=\left|T_{n}^{1}\right|+o\left(n^{2}\right)$ and $\left|E_{n}^{1}\right|=\left|E_{n}\right|+o\left(n^{2}\right)$ we obtain the claim. 
From Claims 1 and 2 it follows that, for $n \in M$,

$$
\left|E_{n}\right|-\lambda(n)\left|T_{n}\right| \leq\left(1-\frac{\lambda(n)}{2}\right)\left|E_{n}\right|+o\left(n^{2}\right) \leq \frac{2-\lambda(n)}{8} n^{2}+o\left(n^{2}\right),
$$

contradicting our assumption (26).

Corollary 5. Let $\operatorname{BLYM}(n)$ be the minimum BLYM-value of a maximal antichain in $\left(\begin{array}{c}{[n]} \\ 2\end{array}\right) \cup\left(\begin{array}{c}{[n]} \\ 3\end{array}\right)$. Then

$$
\lim _{n \rightarrow \infty} \operatorname{BLYM}(n)=\frac{1}{2}
$$

Proof. This follows immediately with

$$
\operatorname{BLYM}(n)=1-\frac{1}{\left(\begin{array}{c}
n \\
2
\end{array}\right)} \varphi_{\lambda}(n),
$$

where $\lambda(n)=3 /(n-2)$.

Corollary 6. Let $\operatorname{vol}(n)$ be the minimum volume of a maximal antichain in $\left(\begin{array}{c}{[n]} \\ 2\end{array}\right) \cup\left(\begin{array}{c}{[n]} \\ 3\end{array}\right)$. Then

$$
\operatorname{vol}(n)=7 n^{2} / 8+o\left(n^{2}\right)
$$

Proof. This follows immediately with

$$
\operatorname{vol}(n)=2\left(\left(\begin{array}{l}
n \\
2
\end{array}\right)-\varphi_{\lambda}(n)\right),
$$

where $\lambda(n)=3 / 2$.

\section{The case $\lambda \geq 2$}

For $\lambda>2$, every T-graph with the maximal value of $|E|-\lambda|T|$ has the property that every edge is contained in exactly one triangle. This is true because otherwise we could increase the value of $|E|-\lambda|T|$ by deleting edges. For $\lambda=2$ there is an optimal T-graph with $D(x y)=1$ for every $x y \in E$, since by deleting edges any T-graph can be transformed into one with this property without changing the value of the objective function. So for this section we suppose that $D(x y)=1$ for every $x y \in E$. Then we have $|T|=\frac{1}{3}|E|$, and the problem is to maximize $|E|$ or $|T|$. As observed in the last section this is equivalent to the $(6,3)$-problem and we obtain $|T|=o\left(n^{2}\right)$. On the other hand, Ruzsa and Szemerédi [8] give an explicit construction which yields, for sufficiently large $n$,

$$
|T| \geq \frac{1}{100} r_{3}(n) n
$$

where $r_{3}(n)$ is the maximal cardinality of a set of positive integers less than $n$ containing no three numbers in an arithmetic progression. According to a result of Behrend [1], for every positive constant $c$ we have $r_{3}(n) \geq n^{1-\frac{c}{\log n}}$ for large enough $n$, hence

$$
|T| \geq \frac{1}{100} n^{2-\frac{c}{\log n}}
$$


Below we describe some optimal constructions for small $n$. For this we need another upper bound on $|T|$ which is worse than $o\left(n^{2}\right)$ but more convenient for showing the optimality of our constructions.

Theorem 7. Suppose $D(x y)=1$ for every $x y \in E$. Then

$$
|T| \leq \begin{cases}\left\lfloor\frac{n(n+3)}{18}\right\rfloor & \text { if } n \text { is odd } \\ \left\lfloor\frac{n(n+2)}{18}\right\rfloor & \text { if } n \text { is even. }\end{cases}
$$

Proof. From $D(x y)=1$ for all $x y \in E$ it follows that, for all $x \in V$,

$$
D(x)=\frac{d(x)}{2}
$$

In particular, $d(x)$ is even for every $x \in V$. Fix some $x \in V$. Since $D(x y)=1$ for every $y \in N(x)$, the subgraph induced by $N(x)$ is a matching of cardinality $d(x) / 2$. For every edge $y z \in E$ with $x y z \in T$ and for every vertex $w \in V \backslash \bar{N}(x)$, from $D(y z)=1$ it follows that

$$
y w \in E \Rightarrow z w \notin E \text { and } \quad z w \in E \Rightarrow y z \notin E .
$$

Recalling that $d(y)$ is even for any $y \in V$, this implies, for any $y z \in E$ with $x y z \in T$,

$$
\begin{gathered}
d(y)+d(z) \leq n-d(x)+3 \quad \text { if } n \text { is odd, and } \\
d(y)+d(z) \leq n-d(x)+2 \quad \text { if } n \text { is even. }
\end{gathered}
$$

Now let $n$ be odd. The even $n$ case is treated analogously. Summing up (27) over the edges $y z$ with $x y z \in T$ we obtain

$$
\sum_{y \in N(x)} d(y) \leq \frac{d(x)}{2}(n-d(x)+3),
$$

Summing up (28) over $x \in V$ yields

$$
\sum_{y \in V} d(y)^{2} \leq(n+3)|E|-\frac{1}{2} \sum_{x \in V} d(x)^{2}
$$

or

$$
\frac{3}{2} \sum_{x \in V} d(x)^{2} \leq(n+3)|E| .
$$

Now the quadratic-arithmetic mean inequality implies

$$
\sum_{x \in V} d(x)^{2} \geq \frac{4|E|^{2}}{n}
$$


and so

$$
|E| \leq \frac{n(n+3)}{6} \quad \text { and } \quad|T| \leq \frac{n(n+3)}{18}
$$

For $n \equiv 3(\bmod 6)$ we can give a complete list of the cases where equality occurs in Theorem 7. These optimal constructions can be interpreted as generalized quadrangles (GQ). For positive integers $s$ and $t$, a $G Q(s, t)$ is an incidence structure $(P, L, I)$, where $P$ and $L$ are disjoint sets of $(s+1)(s t+1)$ points and $(t+1)(s t+1)$ lines, respectively, and $I$ is a symmetric point-line incidence relation satisfying the following axioms:

1. Each point is incident with $1+t$ lines, and two distinct points are incident with at most one line.

2. Each line is incident with $1+s$ points and two distinct lines are incident with at most one point.

3. If $x$ is a point and $l$ is a line not incident with $x$, then there is a unique pair $\left(y, l^{\prime}\right) \in P \times L$ for which $x I l^{\prime}, y I l$ and $y I l^{\prime}$.

For more information on GQs we refer to Payne and Thas [9] .

Theorem 8. Suppose $G$ is a T-graph with $D(x y)=1$ for every $x y \in E$ and $|E|=n(n+$ $3) / 6$. Then $n=3$ or $(V, T)$ is a $G Q(2,(n-3) / 6)$. Conversely, from any $G Q(2,(n-3) / 6)$ with point set $V, 3<|V|=n \equiv 3(\bmod 6)$, and line set $T$, we obtain a T-graph $G=(V, E)$ with $D(x y)=1$ for every $x y \in E$ and $|E|=n(n+3) / 6$ by putting

$$
E=\{x y: \exists l \in T x I l \text { and } y I l\} .
$$

Proof. First, let $G$ be a T-graph with $D(x y)=1$ for every $x y \in E$ and assume $|E|=n(n+3) / 6$. By Theorem $7, n \equiv 3(\bmod 6)$. Using the fact that we need equality in the proof of Theorem 7 , it is easy too see that $(V, T)$ with the natural incidence relation satisfies all the conditions for a $G Q(2,(n-3) / 6)$. Conversely, assume we have a $G Q(2,(n-3) / 6)$ on $n$ points, denoted by $(V, T)$ and let $E$ be defined by (29). It follows that $G=(V, E)$ is a T-graph with $D(x y)=1$ for every $x y \in E$. Since every point is incident with $(n+3) / 6$ lines and every line yields two edges incident with $x$, we obtain $d(x)=(n+3) / 3$ for all $x \in V$, hence $|E|=n(n+3) / 6$.

Two necessary conditions for the existence of a $G Q(s, t)$ are (see [9])

- $s, t>1 \Rightarrow s \leq t^{2}$ and $t \leq s^{2}$,

- $s+t$ divides $s t(s+1)(t+1)$.

So a $G Q(2, t)$ can exist only for $t \in\{1,2,4\}$. Indeed, such GQs on $6 t+3$ vertices do exist (the classical GQs $Q(d, 2)$ for $d=3,4,5[9]$ ), hence we obtain

Corollary 9. There is a T-graph $G$ with $D(x y)=1$ for every $x y \in E$ and $|E|=\frac{n(n+3)}{6}$ iff $n \in\{3,9,15,27\}$. 


\section{Open problems}

1. Is it true that

$$
\varphi_{\lambda}(n)=\frac{2-\lambda(n)}{8} n^{2}+O(n)
$$

holds for $\lambda: \mathbb{N} \mapsto(0,2)$ ?

If so, does our standard construction $K_{r, n-r}^{+}$with $r=n / 2+o(n)$ give the correct linear term?

2. Generalize Theorems 12 and Corollaries 5, 6 to maximal antichains $\mathcal{A} \subseteq\left(\begin{array}{c}{[n]} \\ k-1\end{array}\right) \cup\left(\begin{array}{c}{[n]} \\ k\end{array}\right)$, $k>3$, or to $\mathcal{A} \subseteq\left(\begin{array}{c}{[n]} \\ 2\end{array}\right) \cup\left(\begin{array}{c}{[n]} \\ k\end{array}\right), k>3$.

\section{References}

[1] F. Behrend. On sets of integers which contain no three terms in arithmetical progression. Proc. Nat. Acad. Sci. USA, 32 (1946), 331-332.

[2] J.R. Griggs. Personal communication. 2004.

[3] S. Hartmann, U. Leck and I.T. Roberts. Squashed full flat antichains of minimum weight. Forthcoming.

[4] Á. Kisvölcsey. Flattening antichains. Combinatorica, 308 (2008), no. 11, 2247-2260.

[5] P. Lieby. Extremal problems in finite sets. PhD thesis, Northern Territory University (Australia), 1999.

[6] P. Lieby. Antichains on three levels. Electron. J. Combin., 11 (2004), \#R50.

[7] J.W. Moon and L. Moser. On a problem of Turán. Publ. Math. Inst. Hungar. Acd. Sci., 7 (1962), 283-286.

[8] I.Z. Ruzsa and E. Szemerédi. Triple systems with no six points carrying three triangles. Combinatorics, Keszthely 1976. Colloq. Math. János Bolyai, 18 (1978), 939-945.

[9] S.E. Payne and J.A. Thas. Finite generalized quadrangles. Pitman, 1984. 